\title{
Removal of Fixed-valued Impulse Noise based on Probability of Existence of the Image Pixel
}

\author{
Ali Awad \\ Faculty of Engineering and Information Technology, Alazhar University, Gaza, Palestine
}

\begin{tabular}{l}
\hline \hline Article Info \\
\hline Article history: \\
Received Sep 27, 2017 \\
Revised Jan 20, 2018 \\
Accepted Feb 21, 2018 \\
\hline
\end{tabular}

\section{Keyword:}

Fixed value Image denoising Impulse noise Noise removal Probability of existance

\begin{abstract}
This paper proposes a new approach for restoring images distorted by fixedvalued impulse noise. The detection process is based on finding the probability of existence of the image pixel. Extensive investigations indicate that the probability of existence of a pixel in an original image is bounded and has a maximum limit. The tested pixel is judged as original if it has probability of existence less than the threshold boundary. In many tested images, the proposed method indicates that the noisy pixels are detected efficiently. Moreover, this method is very fast, easy to implement and has an outstanding performance when compared with other well-known methods. Therefore, if the proposed filter is added as a preliminary stage to many filters, the final results will be improved.
\end{abstract}

Copyright $\odot 2018$ Institute of Advanced Engineering and Science. All rights reserved.

\section{Corresponding Author:}

Ali Awad,

Faculty of Engineering and Information Technology,

Alazhar University,

Gaza, Palestine.

Email: aawad@alumi.stevens.edu

\section{INTRODUCTION}

The Digital image may be corrupted by different types of noise such as Gaussian, impulse, sparkle, and many others. Impulse noise usually corrupts the digital images due to errors produced from the noisy sensors or communication channel. It has a short duration and a sharp length. If impulse noise corrupts group of pixels in the original image, then they will be replaced by the impulse values, and the other pixels remains intact. As a result, the new image will have two types of pixels, the firt one is the noisy pixels $\boldsymbol{n}$ 's that represent the impulse noise and the other type is the original pixels $s$ 's. Besides, there are two types of impulse noise, the first one is fixed valued impulse noise and the other one is random valued impulse noise. In the fixed type, the noise may take one or more value from the range of [0,255]. Example of this type is salt and pepper noise. In which, the salt noise takes a value equal to 255 and the pepper noise takes a value equal to 0 . In random type, the noise may take any value in the dynamic range of $[0,255]$. In this paper, fixed valued impulse noise is investigated and the original image is artificially corrupted, either by one, two, or three fixed values. Impulse noise $\boldsymbol{n}(\boldsymbol{i}, \boldsymbol{j})$ located at $\boldsymbol{i}, \boldsymbol{j}$ in the noisy image is modeled by a binary Bernoulli process $\boldsymbol{b}(\boldsymbol{i j})$ [1].In which $\boldsymbol{b}(\boldsymbol{i}, \boldsymbol{j})$ equals to " $\boldsymbol{I}$ " with a probability $\boldsymbol{p}$ and equals to " $\boldsymbol{0}$ " with a probability $\boldsymbol{1}-\boldsymbol{p}$. Thus, the noisy pixel $\boldsymbol{x}(\boldsymbol{i}, \boldsymbol{j})$ is expressed as:

$$
x(i, j)=b(i, j) n(i, j)+(1-b(i, j)) s(i, j)
$$

The challenge here is to restore the noisy image in such a way that preserve the fine details of the original image in the recovered version. The famous filter that used to suppress the impulse noise is the median filter [2]. Median filter replaces the tested pixel with the median value of a pre-defined filtering window centered at the tested pixel. The drawback of the median filter is that it replaces every pixel in the 
image regardless if the tested pixel is noisy or not. Thus, many of the original pixels will be replaced by noisy values. As a result, the resultant restored image looks burred, and lose many original details. For enhancing the performance of the median filter, family of median-based filters are proposed. Among them are weighted median filter [3], and center weighted median filter [4]. In both, more weight is given to the pixels that are most likely original. Other median based filters are tri-state median filter [5], progressive-switching median filter [6], multi-state median filter [7], and pixel-wise MAD-based (PWMAD) filter [8].

These filters are decision-based filters used for preserving more image details by firstly detecting the noisy pixels. If the pixel is detected as noisy, then it is restored. To effectively restore the highly corrupted images, some novel switching-based filters have been proposed such as switching median filter with boundary discriminative noise detection BDND) [9], median-type noise detectors and detail-preserving regularization [10], and an iterative adaptive fuzzy filter based on two stages technique [11] and many others as [12]-[14].The authors in [15]-[17] proposed a new method based on the value of the noisy pixel and the pixels in the tested window. The drawback of these filters is that they provide rstored images with poor visual quality at high noise rates. Recently, many methods based on spare representation are used in literature as the ones mentioned in [18]-[21]. Unfortunately, these methods improve the results at high noise rates but at the expense of image details or computational complexity. In his paper, we assume that the fixed valued Impulse noise may take one, two, or three values each with different probability as:

$$
x(i, j)=\left\{\begin{array}{l}
v 1 \text { withprobbiyit } p 1 \\
v 2 \text { withprobbiyt p2 } \\
v 3 \text { withprobbilit p3 } \\
s(i, j) \text { withprobbiyi }-p
\end{array}\right.
$$

Where, $\boldsymbol{p} \mathbf{1}+\boldsymbol{p} \mathbf{2}+\boldsymbol{p} \mathbf{3}=\boldsymbol{p}$. If the image is corrupted only by one value, then let $\boldsymbol{p} \mathbf{2}=\boldsymbol{p} \mathbf{3}=\mathbf{0}$. If the image is corrupted by two values each with probability of $\mathbf{5 0 \%}$, then let $\mathbf{p 3}=\mathbf{0}$. Thus, a new technique is used in this paper for detecting the noisy pixel based on the probability of existence of the tested pixel. More specifically, we should find the probability of existence of the tested pixel in order to be detected as original or noisy pixel. Any pixel is detected as noisy pixel if its probability of existence is more than a specific threshold. The proposed idea is unique and delivers superior and an outstanding results as shown in the following sections. The adopted idea does not depend on the surrounding pixels if they are original or not, and does not depend on the mean, median, or any other parameter for the image. It depends on independent factor which determines the presence of any value in the image.

\section{RESEARCH METHOD}

The proposed algorithm is divided into two parts the first part explains the detection process and the second part demonstrates the recovery process as shown below.

\subsection{Detection process}

From the literature, it is clear that many of the researchers make use the correlation between the pixels in the filtering window to detect the noisy pixels. In this paper, a new principle based on the probability of existence is proposed in the detection process. In the sense that, the repetitions of each pixel in the image is calculated, to help judging whether the tested pixel is an original pixel or not. To this end, we gather all the pixels that have equal values in separated groups. The number of the pixels in each group is the figure that we seek for. To illustrate the detection process, let $\boldsymbol{X}$ denotes to a noisy image of $\boldsymbol{m} \times \boldsymbol{l}$ size. The pixels that have density values equal to $\boldsymbol{v}_{\boldsymbol{i}}$ are collected in a separate group $\boldsymbol{g}_{\boldsymbol{i}}, \boldsymbol{i}=\mathbf{1}: \boldsymbol{K}$, where $\boldsymbol{K}$ is the number of the groups. The number of the pixels in the group $\boldsymbol{g}_{\boldsymbol{i}}$ is denoted by $\boldsymbol{n}_{\boldsymbol{i}}$. Thus, the group $\boldsymbol{g}_{\boldsymbol{i}, \boldsymbol{n} \boldsymbol{i}}$ is defined as:

$$
g_{i n i}=\left[v_{i, 1} v_{i, 2} v_{i, 3} \quad \ldots \ldots v_{i n i}\right]
$$

Two matrices from (3) are defined; matrix $\boldsymbol{V}$ that contains one value from each group and matrix $\boldsymbol{N}$ that encloses the number $\boldsymbol{n}_{\boldsymbol{i}}$ of the corresponding value $\boldsymbol{v}_{\mathrm{i}}$. Therefore, the matrices $\boldsymbol{V}$ and $\boldsymbol{N}$ are described as:

$$
\begin{aligned}
& V=\left[\begin{array}{lllll}
v_{1} & v_{2} & v_{3} & \ldots & v_{K}
\end{array}\right] \\
& n=\left[\begin{array}{lllll}
n_{1} & n_{2} & n_{3} & \ldots & n_{K}
\end{array}\right]
\end{aligned}
$$


The probability of existence $\boldsymbol{p}_{r}$ of a value $\boldsymbol{v}_{\boldsymbol{r}}, \boldsymbol{l} \leq \boldsymbol{r} \leq \boldsymbol{K}$, is defined as:

$$
p_{r}=\frac{n_{r}}{l \times m}
$$

Matrix $\boldsymbol{P}$ that includes the probability of existence for all image values is defined as:

$$
P=\left[\begin{array}{lllll}
p_{1} & p_{2} & p_{3} & \ldots & \ldots \\
p_{K}
\end{array}\right]
$$

Define a new vector $\overline{\boldsymbol{P}}$ to include all the probabilities that are greater than a threshold value $\boldsymbol{p}_{t h}$ as:

$$
\left.\bar{P}=\sum p_{i}, p_{i}>p_{t h}, 1 \leq i \leq M, M<K\right)
$$

From (8) one can conclude that:

$$
\overline{\bar{P}}=1-\sum_{i=1}^{M} p_{i}==\sum_{i=M+1}^{K} p_{i}=1-\bar{P}
$$

Where $\overline{\bar{P}}$ represents the accumulative probability of all the probabilities that are less than $\boldsymbol{p}_{\boldsymbol{t} \boldsymbol{h}}$ and it is usually small value as shown in Table 1 . Also, it is clear that,

$$
\begin{aligned}
& \sum_{i=1}^{M} p_{i}>M P_{t h} \leftrightarrow \sum_{i=1}^{M} p_{i}-M P_{t h}>0 \\
& 1-\overline{\bar{P}}-M P_{t h}>0 \\
& P_{t h}<\frac{1}{M}-\frac{\overline{\bar{P}}}{M}
\end{aligned}
$$

Let $\frac{\overline{\bar{P}}}{\boldsymbol{M}}=0$ then the maximum bound for $\boldsymbol{p}_{\boldsymbol{t}}$ is less than $\frac{\boldsymbol{1}}{\boldsymbol{M}}$. Also, at highly corrupted images, the ratio $\frac{\overline{\overline{\boldsymbol{P}}}}{\boldsymbol{M}}$ is small because the noise rate or the probability of existence of the noisy pixel is high with respect to that of the original pixel, $\overline{\bar{P}}<<$, thus this ratio can be neglected and the value of $\boldsymbol{p}_{\boldsymbol{t h}}$ becomes as:

$$
\boldsymbol{O}<\boldsymbol{P}_{\mathrm{th}}<\frac{1}{M}
$$

From Table 1, one can observe that for a sample of $\mathbf{2 4}$ images, $\boldsymbol{p}_{\boldsymbol{t} \boldsymbol{h}}$ in any original image does not exceed than $4 \%$. That means, we could not find a pixel in any original image with probability of existence equal to or more than 4\%. It is clear from Figure 1 and Table 1, for Lena image, the pixel of the value 224 has the maximum probability of existence equal to $\mathbf{1 . 5 \%}$. Thus, the pixel in the noisy image that has probability of existence $\boldsymbol{p}_{\boldsymbol{r}}$ more than $\boldsymbol{p}_{\boldsymbol{t} \boldsymbol{h}}$ is considered noisy pixel, as described in Figure 2. Consequently, if $\boldsymbol{p}_{\boldsymbol{r}}>\boldsymbol{p}_{\boldsymbol{t} \boldsymbol{h}}$, then the pixel value $v_{\boldsymbol{r}}$ at position $\boldsymbol{r}$ in matrix $\boldsymbol{V}$ is deemed noisy pixel as:

$$
\text { if } p_{r}=\frac{n_{r}}{l \times m}>p_{t h} \text { then } v_{r} \text { is noisy vlue }
$$

At the end, the value $\boldsymbol{v}_{\boldsymbol{r}}$ is flagged as $\boldsymbol{l}$ in the corresponding position in an image $\boldsymbol{F}$ of $\boldsymbol{z}$ ero elements. As an example, noisy image corrupted with $\mathbf{8 0 \%}$ salt and pepper noise rate has two noisy pixels each has probability of existence $\boldsymbol{p r}=\mathbf{4 0 \%}$ which is more than $\boldsymbol{p}_{\boldsymbol{t h}}$. Thus, we have only two values, i.e., $\boldsymbol{M}=\mathbf{2}$, that satisfy the condition in (14). During this paper, we consider $\boldsymbol{p}_{\boldsymbol{t h}}=\mathbf{5 \%}$ which is less than $\mathbf{1 / M}$, as indicated in 13 as:

$$
P_{r}=\frac{\text { noiserate }}{2}>5 \%<\frac{1}{2}
$$


The following example represents the probability of existence for original and noisy values, let $P$ and $V$ as:

$$
\begin{aligned}
& P=\left[\begin{array}{llllllllll}
0.03 & 0.03 & 0.02 & 0.02 & 0.4 & 0.03 & 0.03 & 0.02 & 0.02 & 0.4
\end{array}\right] \\
& V=\left[\begin{array}{llllllllll}
10 & 20 & 30 & 40 & 5 & 16 & 160 & 40 & 111 & 255
\end{array}\right]
\end{aligned}
$$

Then, it is clear that $\boldsymbol{P}_{5}=\mathbf{4 0 \%}, \boldsymbol{P}_{10}=\mathbf{4 0 \%}$, and probability of original values is $\overline{\bar{P}}=\mathbf{1}-\mathbf{0 . 8}=\mathbf{0 . 2}$ or $\mathbf{2 0 \%}$.

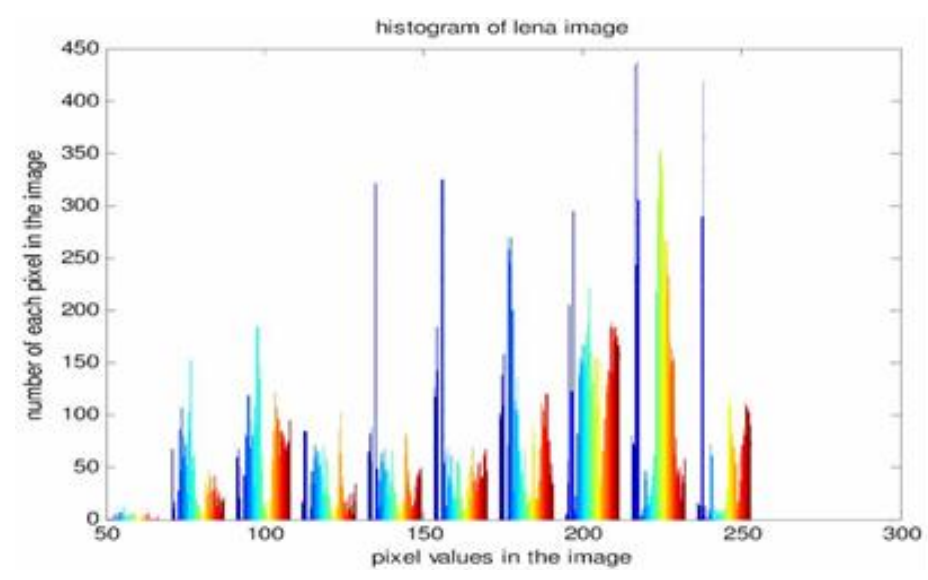

Figure 1. Illustrates the replication of each pixel in Lena image

Table 1. Maximum Probability of Existence in different known Images

\begin{tabular}{lclclc}
\hline \multicolumn{1}{c}{ Image (value) } & ratio\% & \multicolumn{2}{c}{ Image (value) } & ratio\% & \multicolumn{2}{c}{ Image (value) } & ratio\% \\
\hline Lena (224) & 1.5 & Lake (150) & 1.31 & Truck (129) & 2.35 \\
Bridge (82) & 0.87 & Moon (134) & 1.85 & Tank (149) & 2.91 \\
Pepper (199) & 1.48 & Airplane (207) & 3.06 & Bear (0) & 3.05 \\
Boot (147) & 2.0 & Elaine (133) & 0.90 & Opera (144) & 1.89 \\
Pentagon (130) & 1.81 & Gun (154) & 1.42 & Paper machine (206) & 2.36 \\
Einstein (44 & 2.42 & Water (106) & 1.41 & Couple (3) & 3.38 \\
Watch (24) & 3.7 & Brandyrose (154) & 2.28 & Waterfall (117) & 2.04 \\
\hline
\end{tabular}

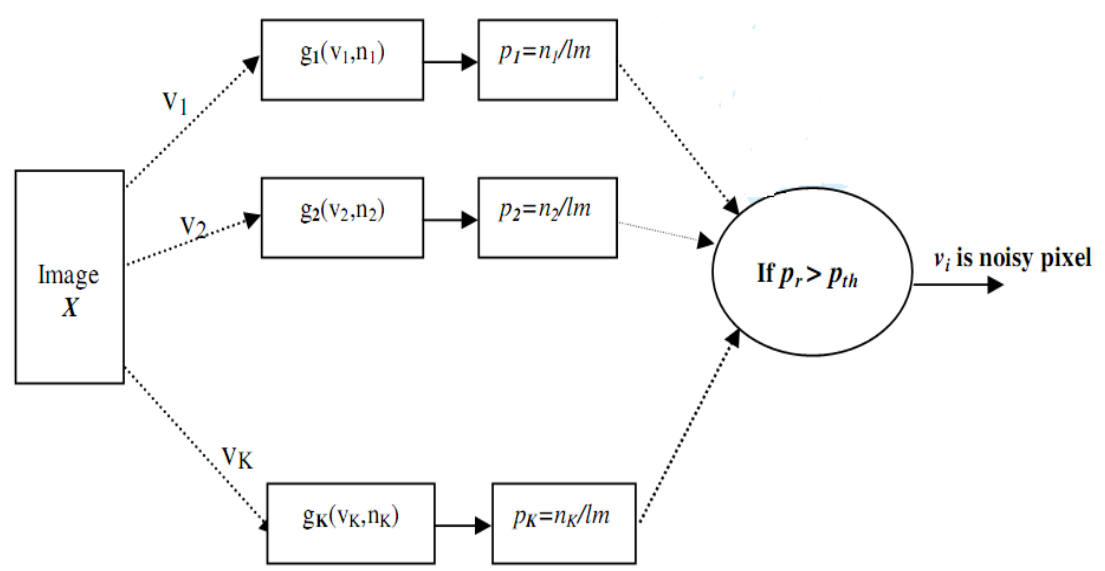

Figure 2. Illustrates the block diagram of the proposed detection technique

\subsection{Restoration process}

A pixel $\boldsymbol{x}_{i j}$ flagged as $\boldsymbol{f}_{i j}=\boldsymbol{I}$ in $\boldsymbol{F}$ is replaced by the median value of its neighboring pixels $\boldsymbol{Y}_{i j}$. Any pixel detected as noisy pixel and belong to $\boldsymbol{Y}_{i j}$ is excluded and the median is taken for the rest good pixels. This process is implemented recursively, in the sense that the current restored pixel is used in the restoration of the next noisy pixel in the subsequent windows. A small window $\boldsymbol{W}$ of $\mathbf{3} \times \mathbf{3}$ size is enough to be used in the

Removal of Fixed-Valued Impulse Noise based on Probability of Existence of the Image Pixel (Ali Awad) 
restoration process, but in the case of heavily corrupted image, noise rate $>70 \%$, a window of $5 \times 5$ size or more is used. The neighboring pixels $\boldsymbol{Y}_{i j}$ and the restored version $\boldsymbol{x}_{\boldsymbol{i} j, \boldsymbol{r e s t}}$ of the noisy pixel $\boldsymbol{x}_{\boldsymbol{i} j}$ are defined as:

$$
\begin{gathered}
Y_{i j}=\left\{x_{i-s, j-t} \times f_{i-s, j-t} \mid s, t \in W\right\} \\
x_{i j, r e s t}=\operatorname{median}\left(Y_{i j}\right)
\end{gathered}
$$

The new method has the ability to detect the noisy pixel with zero error in large number of images. Thus, more powerful restoration process increases the performance of the proposed method and the visual quality of the recovered images.

\section{RESULTS AND ANALYSIS}

The proposed algorithm is compared with different known methods in terms of visual quality, processing time, and peak signal to noise ratio, PSNR. Note that the values of PSNR demonstrate the degree of sameness or closeness between the restored values and the original ones.Tested images are corrupted artificially with different noisy values. They are corrupted either with one, two, or three noisy values based on the MATLAB "imnoise" command. The strength of the proposed algorithm is showm clearly through its performance at high noise rates. Most of the known methods perform well at low noise rates, but the problem when the noise rate becomes $>40 \%$. In this case, many of the well-known methods detect wrongly the true pixels. Therefore, maintaining the fine image details while detecting the noisy pixels is a challenge task. The advantage of the adopted algorithm is that it depends on a novel and unique feature explored during this research.This feature denotes that the structure and the view of any real image should have pixels its values fluctuate regularly and with some correlation.Based to this feature, the proposed method is capable of detecting all the noisy pixels correctly with a $100 \%$ accuracy, either at high or low noise rates and for large numbers of images. In the proposed approach, it is not necessary to classify the pixels as original, noisy, or most likely noisy through the detection and restoration processes as in many known methods. More specifically, the principle of the most known methods depends on one or more parameter or value such as the mean, median, standard deviation, noisy value, distance between the neighboring pixel, window size, correlation among the pixels, or to one or more threshold value.

Unfortunately, these parameters or values fail to detect the noisy pixels, particurly at high noise rates. Because most of the pixels at this noise rates are noisy and therefore they involve in the detection process. Besides, it is difficult to estimate the original value of any pixel if most of their surrounding pixels are noisy. On the other hand, the principle of the new approach depends only on the weight of each pixel in the image.In other words, the assumed method indicates that the existance of any original pixel in any image is bounded and not exceeds a specific limit. But, the problem is how we can determine this limit to be a benchmark for detecting the noisy pixels. It is shown in Table 1and Figure 1 that the probability of finding any pixel in many original images is very small and not exceeds than $4 \%$. Thus, the detection process states that if any pixel has probability of existence $\boldsymbol{p}_{\boldsymbol{r}} \geq$ threshold probability $\boldsymbol{p}_{\boldsymbol{t}}$, then it is considered noisy pixel. Threshold probability that used during the simulation is $\boldsymbol{p}_{\boldsymbol{t h}} \mathbf{= 5 \%}$. To demonstrate the power of the proposed method, extensive of experiments are performed and the results are shown numerically and visually through the following tables and figures.

It is obvious from the results shown in Table 2, Table 3, and Table 4 that the proposed method achieves superior results comparing with the other known methods. It is also apparent from these tables that the new method provides the best PSNR values either at low or high noise rates in restoring different images corrupted with 0 and 255 noisy values. It is notable that, the performance of the proposed method in restoring corrupted Lena image is better than its performance in restoring bridge and baboon images. The reason is that the neighboring pixels in the bridge and baboon images are not similar enough to each other. As a result, the restored pixel during the recovery process does not reflect the optimum estimated value. Thus, the same detection method with more powerful recovery process would deliver better performance. Table 5 illustrates the performance of different methods in term of PSNR values when Lena image is corrupted by two different values 20 and 200. It is clear that the new method delivers superior results at low and high noise rates. Figure 3 compares the performance of the best two known methods with the proposed one in restoring corrupted Lena image with $90 \%, 70 \%$, and $50 \%$ noise rates. Figure 4, demonstrations the performance of the proposed technique in restoring corrupted versions of boat, bridge, and baboon images. They corrupted at $60 \%$ noise rate by the salt and pepper noise. It is notable that the proposed one outperforms clearly the others.

Int J Elec \& Comp Eng, Vol. 8, No. 4, August 2018 : 2106 - 2114 
Table 2. Comparison for Restoring Corrupted Lena Image with 0 and 255 in Terms of PSNR (dB)

\begin{tabular}{lccccccccc}
\hline $\begin{array}{c}\text { Method/ } \\
\text { noise rate }\end{array}$ & $10 \%$ & $20 \%$ & $30 \%$ & $40 \%$ & $50 \%$ & $60 \%$ & $70 \%$ & $80 \%$ & $90 \%$ \\
\hline PSM [6] & 33.06 & 30.00 & 27.91 & 26.20 & 23.92 & 21.63 & 19.12 & 15.48 & 11.13 \\
ACWMF [4] & 37.21 & 34.75 & 31.87 & 28.54 & 24.95 & 20.62 & 16.16 & 12.32 & 8.25 \\
PWMAD [8] & 35.94 & 26.59 & 20.23 & 16.05 & 13.00 & 10.64 & 8.73 & 7.18 & 5.89 \\
TSM [5] & 25.55 & 22.56 & 18.67 & 15.01 & 12.14 & 9.82 & 7.95 & 6.51 & 5.43 \\
MSM [7] & 38.24 & 30.49 & 24.75 & 19.46 & 15.50 & 12.35 & 9.60 & 7.44 & 5.95 \\
NEW & 38.34 & 35.65 & 34.16 & 33.00 & 32.013 & 31.19 & 30.38 & 29.24 & 26.76 \\
\hline
\end{tabular}

Table 3. Comparison for Restoring Corrupted Bridge Image with 0 and 255 in Terms of PSNR (dB)

\begin{tabular}{lccccccccc}
\hline $\begin{array}{c}\text { Method/ } \\
\text { noise rate }\end{array}$ & $10 \%$ & $20 \%$ & $30 \%$ & $40 \%$ & $50 \%$ & $60 \%$ & $70 \%$ & $80 \%$ & $90 \%$ \\
\hline PSM [6] & 28.86 & 26.75 & 24.76 & 22.67 & 20.88 & 18.92 & 16.78 & 14.26 & 10.45 \\
ACWMF [4] & 27.27 & 26.12 & 24.71 & 23.06 & 21.08 & 18.38 & 15.48 & 12.13 & 8.30 \\
PWMAD [8] & 28.90 & 24.05 & 19.06 & 15.38 & 12.70 & 10.63 & 8.94 & 7.48 & 6.24 \\
TSM [5] & 20.92 & 19.95 & 17.50 & 14.76 & 12.18 & 9.99 & 8.23 & 6.85 & 5.84 \\
MSM [7] & 28.36 & 26.20 & 23.01 & 18.84 & 15.43 & 12.26 & 9.70 & 7.70 & 6.31 \\
NEW & 34.81 & 31.74 & 29.76 & 28.21 & 26.81 & 25.45 & 23.97 & 22.25 & 19.97 \\
\hline
\end{tabular}

Table 4. Comparison for Restoring Corrupted Babbon Image with 0 and 255 in Terms of PSNR (dB)

\begin{tabular}{lccccccccc}
\hline $\begin{array}{c}\text { Method/ } \\
\text { noise rate }\end{array}$ & $10 \%$ & $20 \%$ & $30 \%$ & $40 \%$ & $50 \%$ & $60 \%$ & $70 \%$ & $80 \%$ & $90 \%$ \\
\hline PSM [6] & 24.17 & 23.25 & 22.46 & 21.67 & 20.72 & 19.69 & 18.23 & 16.21 & 11.88 \\
ACWMF [4] & 23.73 & 23.08 & 22.32 & 21.39 & 19.86 & 17.86 & 15.14 & 11.97 & 8.58 \\
PWMAD [8] & 25.69 & 21.96 & 17.91 & 14.79 & 12.31 & 10.43 & 8.76 & 7.40 & 6.17 \\
TSM [5] & 19.72 & 18.83 & 16.74 & 14.23 & 11.83 & 9.81 & 8.16 & 6.82 & 5.81 \\
MSM [7] & 24.52 & 23.24 & 21.10 & 18.14 & 14.93 & 12.09 & 9.74 & 7.65 & 6.28 \\
NEW & 32.16 & 29.10 & 27.15 & 25.74 & 24.50 & 23.40 & 22.35 & 21.20 & 19.86 \\
\hline
\end{tabular}

Table 5. Comparison for Restoring Corrupted Lena Image with 20 and 200 in Terms of PSNR (dB)

\begin{tabular}{lccccccccc}
\hline $\begin{array}{c}\text { Method/ } \\
\text { noise rate }\end{array}$ & $10 \%$ & $20 \%$ & $30 \%$ & $40 \%$ & $50 \%$ & $60 \%$ & $70 \%$ & $80 \%$ & $90 \%$ \\
\hline PSM [6] & 33.16 & 30.27 & 28.18 & 26.39 & 24.10 & 22.29 & 19.89 & 16.93 & 13.08 \\
ACWMF [4] & 36.52 & 33.69 & 30.39 & 26.68 & 23.06 & 19.97 & 16.81 & 13.25 & 9.79 \\
PWMAD [8] & 36.66 & 28.96 & 22.38 & 18.02 & 14.92 & 12.28 & 10.26 & 8.65 & 7.27 \\
TSM [5] & 25.55 & 23.39 & 19.70 & 16.19 & 13.44 & 11.22 & 9.46 & 8.07 & 6.98 \\
MSM [7] & 36.53 & 31.48 & 25.40 & 20.50 & 16.95 & 13.51 & 11.00 & 8.94 & 7.34 \\
NEW & 42.77 & 39.47 & 37.27 & 35.39 & 33.55 & 31.79 & 30.54 & 29.32 & 26.90 \\
\hline
\end{tabular}

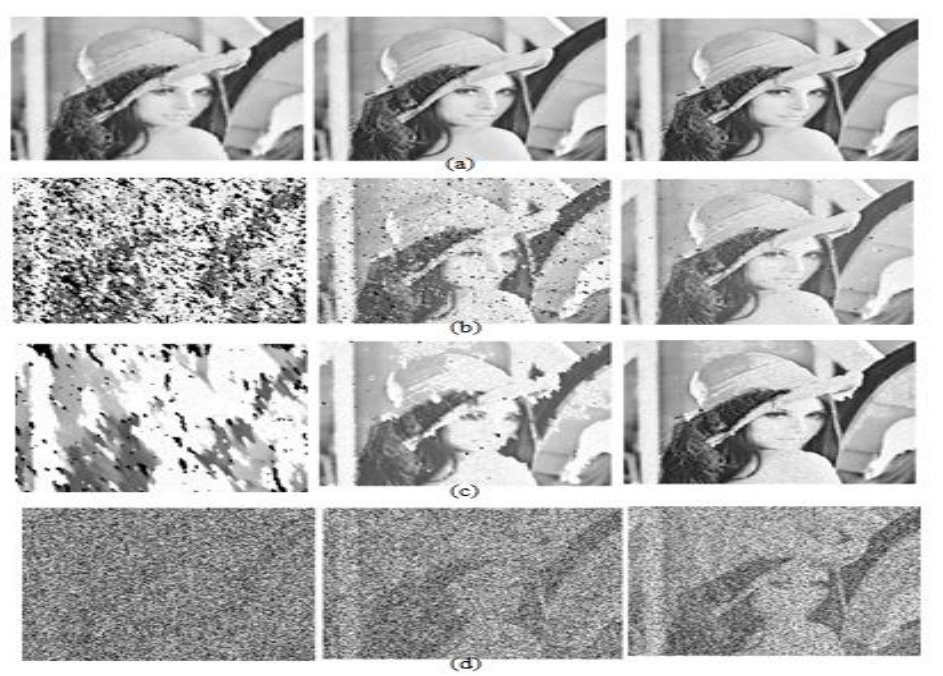

Figure 3. Comparison between existing methods and the proposed one for restoring salt and pepper noise [0,255] corrupted Lena images: (a) New (b) ACWM (c) PSM (d) corrupted Lena images. $1^{\text {st }}, 2^{\text {nd }}$, and $3^{\text {rd }}$ images in row (d) are corrupted by $90 \%, 70$, and $50 \%$ noise rates, respectively 

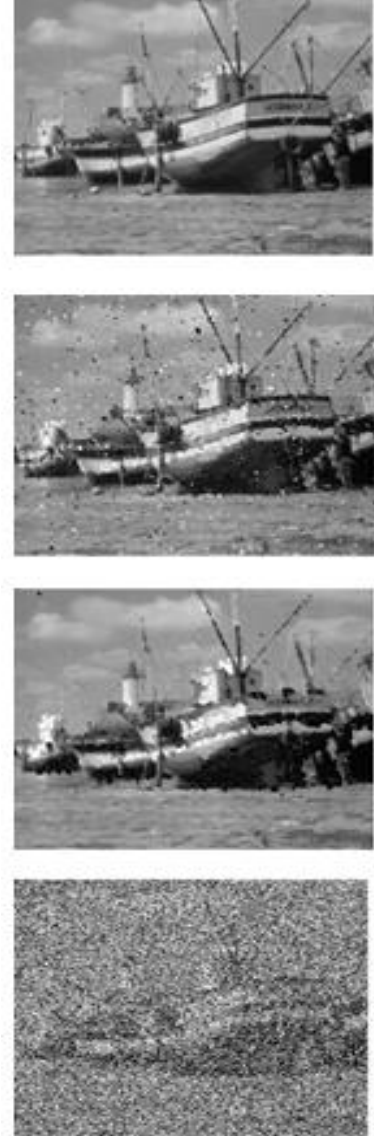

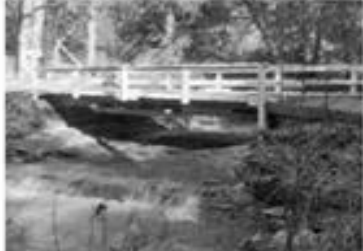

(a)

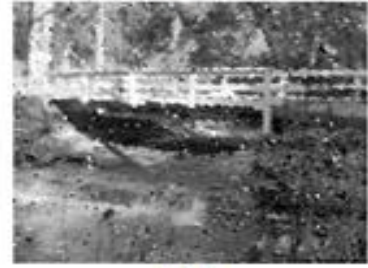

(b)

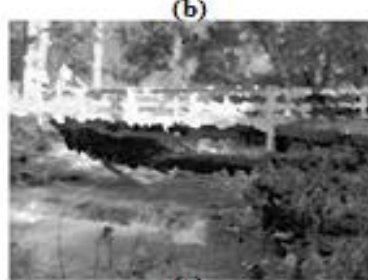

(c)

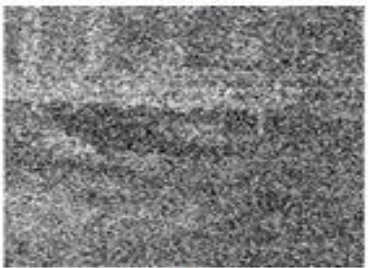

(d)
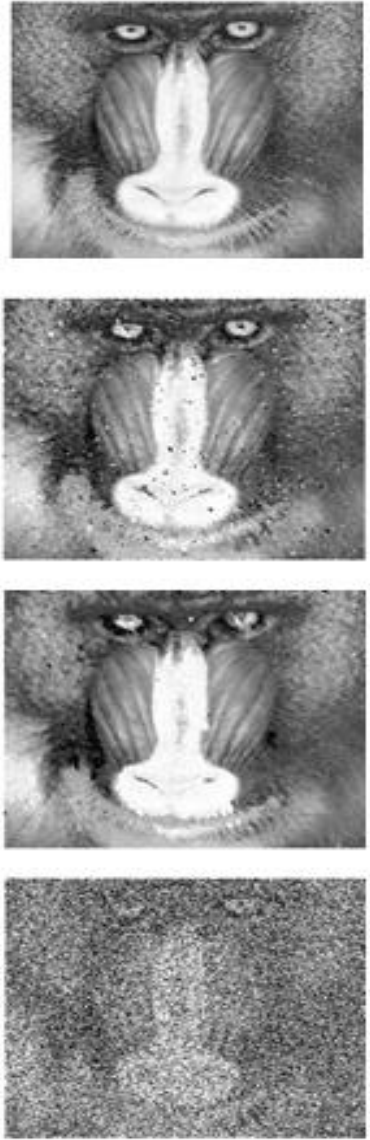

Figure 4. Comparison between existing methods and the proposed one for restoring $60 \%$ salt and pepper noise [0,255] corrupted three differnt images. Corrupted images: (a) New (b) ACWM (c) PSM

(d) corrupted boat, bridge, and baboon images

Figure 5 illustrates the performance of the proposed method in the restoration of $60 \%$ corrupted pepper images either by one, two or three noisy values. It is clear that the proposed method shows superior performance than the others, particularly at $70 \%$ and $90 \%$ noise rates. It is also clear that there are residual noise and blur in the restored images taken from the other methods. Moreover, one can note that the increment in the noisy pixels does not affect the performance of the proposed method. The only thing that shakes its performance is the efficiency of the restoration process in replacing the noisy pixel by an estimated value similar to the original one. Thus, in the restoration process, it is recommended to use a wider filtering window at high noise rates to make sure that the filtering window has an enough number of original or already restored pixels for well-estimate the noisy pixel. Table 6 shows the performance of the new filter in restoring Lena image corrupted with one noisy value (30) at different noisy rates. Table 7 illustrates the performance of different methods in restoring lina image corrupted with three noisy values 0,255 , and 30 with rates of $50 \%, 25 \%$ and $25 \%$, respectively. It is clear that the proposed method is an extraordinary approach in terms of PSNR, and particularly at high noise rates.

The other known methods have difficulty to detect the noisy pixels, mostly at highly corrupted images. The reason is that the other methods almost depend on threshold values based on different parameters such as mean, median, or standard deviation of the surrounding pixels. These parameters almost provide inaccurate values when most of the surrounding pixels are noisy. Figure 6 depicts the consumed time taken by the PSM, ACWMF, and the proposed one in restoring pepper image at different noise rates. It is clear that, the new method outperforms the others in terms of the processing time in seconds. Thus, the proposed filter is simple, converges very fast, and delivers superior results. MATLAB program, CPU of $1.73 \mathrm{GHz}$, and RAM of $1 \mathrm{~GB}$ are used in all the simulated experiments. 

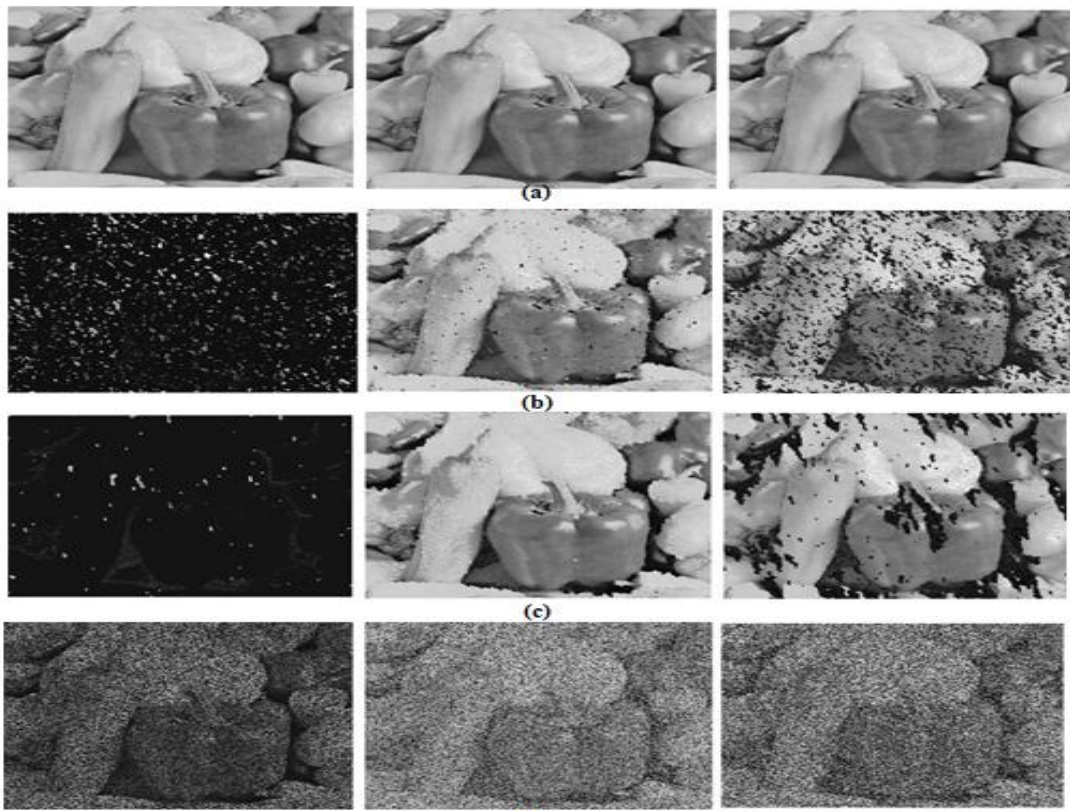

(d)

Figure 5. Comparison between existing methods and the proposed one for restoring corrupted versions of pepper image: row (a) New, row (b) ACWM, row (c) PSM, row (d) corrupted versions of pepper images by

$60 \%$ noise rates. In row (d) first pepper image corrupted by one value [30], $2^{\text {nd }}$ image corrupted by two values $[20,200]$, and $3^{\text {rd }}$ image corrupted by three values $[0,255,30]$, respectively

Table 6. Comparison for Corrupted Lena Image by one value " 30 " in Terms of PSNR (dB)

\begin{tabular}{lccccccccc}
\hline $\begin{array}{c}\text { Method/ } \\
\text { noise rate }\end{array}$ & $10 \%$ & $20 \%$ & $30 \%$ & $40 \%$ & $50 \%$ & $60 \%$ & $70 \%$ & $80 \%$ & $90 \%$ \\
\hline PSM [6] & 37.53 & 33.93 & 29.43 & 20.09 & 6.97 & 4.19 & 4.15 & 4.15 & 4.15 \\
ACWMF [4] & 36.11 & 30.96 & 23.15 & 14.95 & 7.28 & 4.51 & 4.19 & 4.15 & 4.15 \\
PWMAD [8] & 31.90 & 20.09 & 13.63 & 9.63 & 7.11 & 5.52 & 4.64 & 4.24 & 4.15 \\
TSM [5] & 23.77 & 17.29 & 12.38 & 9.21 & 7.07 & 5.67 & 4.79 & 4.32 & 4.16 \\
MSM [7] & 33.50 & 21.95 & 14.88 & 10.14 & 7.05 & 5.32 & 4.50 & 4.21 & 4.15 \\
NEW & 42.99 & 39.56 & 37.28 & 35.40 & 33.66 & 31.88 & 30.65 & 29.38 & 26.78 \\
\hline
\end{tabular}

Table 7. Comparison for Corrupted Lena Image by 0 (50\%), 255 (25\%), and 30 (25\%), in Terms of PSNR $(\mathrm{dB})$

\begin{tabular}{lccccccccc}
\hline $\begin{array}{c}\text { Method/ } \\
\text { noise rate }\end{array}$ & $10 \%$ & $20 \%$ & $30 \%$ & $40 \%$ & $50 \%$ & $60 \%$ & $70 \%$ & $80 \%$ & $90 \%$ \\
\hline PSM [6] & 34.68 & 31.93 & 29.66 & 27.16 & 22.65 & 14.37 & 4.56 & 3.74 & 3.69 \\
ACWMF [4] & 36.93 & 33.91 & 28.96 & 23.25 & 17.05 & 10.68 & 5.81 & 4.46 & 4.18 \\
PWMAD [8] & 35.23 & 24.38 & 17.59 & 13.12 & 10.11 & 7.96 & 6.35 & 5.24 & 4.49 \\
TSM [5] & 25.29 & 21.41 & 16.62 & 12.85 & 10.12 & 8.03 & 6.49 & 5.37 & 4.65 \\
MSM [7] & 36.43 & 28.71 & 21.78 & 16.43 & 12.14 & 8.82 & 6.40 & 5.08 & 4.48 \\
NEW & 39.06 & 37.00 & 35.21 & 33.43 & 31.66 & 29.61 & 30.46 & 29.28 & 26.67 \\
\hline
\end{tabular}

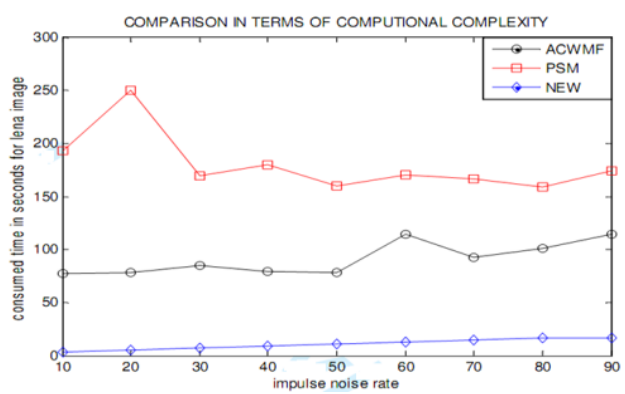

Figure 6. Comparison between different known methods and the new one in terms of computional complexity due to restoring pepper image corrupted at different noise rates 


\section{CONCLUSION}

In this paper, a new filter based on a unique idea is proposed. It has been shown that the presence of any pixel in many tested images is bounded. Thus, the tested pixel is considered noisy pixel if its probability of existence is more than specific threshold. Extensive simulation results prove that the proposed method provide outstanding results at low and high noise rates. The reason is that its detection process does not depend on any correlation between the neighboring pixels which vary from window to window and from region to region. Moreover, the new filter is very fast and easy to implement.

\section{REFERENCES}

[1] N. C. Gallagher and G. L. Wise, "A Theoretical Analysis of the Properties of Median Filters," IEEE Trans .Acoust., Speech and Signal Processing, vol. ASSP-29, pp. 1136-1141, 1981.

[2] A. Muntasa, I. A. Sirajudin, M. H. Purnomo, "Appearance global and local structure fusion for face image recognition", TELKOMNIKA (Telecommunication, Computing, Electronics and Control), 2011, vol. 9, no. 1, pp. $125-132$

[3] L. Yin, et al., "Weighted median filters: A tutorial," IEEE Trans. Circuits Syst II., Analog Digit. Signal Process, vol. 43, no. 3, pp. 157-192, 1996.

[4] T. Chen and H. R. Wu, "Adaptive impulse detection using center-weighted median filters," IEEE Signal Process. Lett, vol. 8, no. 1, pp. 1-3, 2001.

[5] T. Chen, et al., "Tri-state median filter for image denoising," IEEE Trans. Image Process., vol. 8, no. 12, pp. 1834-1838, 1999.

[6] Z. Wang and D. Zhang, "Progressive switching median filter for the removal of impulse noise from highly corrupted images," IEEE Trans. Circuits Syst. II., Analog Digit. Signal Process, vol. 46, no. 1, pp. 78-80, 1999.

[7] T. Chen and H. R. Wu, "Space variant median filters for the restoration of impulse noise corrupted images," IEEE Trans. Circuits Syst. II. Analog Digit. Signal Process, vol. 48, no. 8, pp. 784-789, 2001.

[8] V. Crnojevic, et al., "Advanced impulse detection based on pixel-wise MAD," IEEE Signal Process. Lett, vol. 11, no. 7, pp. 589-592, 2004.

[9] P. E. Ng and K. K. Ma, "A switching median filter with boundary discriminative noise detection for extremely corrupted images," IEEE Trans. Image Process, vol. 15, no. 6, pp. 1506-1516, 2006

[10] R. H. Chan, et al., "Salt-and-Pepper Noise Removal by Median-Type Noise Detectors and Detail-Preserving Regularization," IEEE Trans. Image Process, vol. 14, no. 10, 2005.

[11] F Ahmed and Swagatam, "Removal of High-Density Salt-and-Pepper Noise in Images With an Iterative Adaptive Fuzzy Filter Using Alpha-Trimmed Mean," IEEE Trans on Fuzzy Systems, vol. 22, no. 5, pp. 1352-1358, 2014.

[12] S. W. Jang and M. Jung, "Robust detection of mosaic regions in visual image data," Cluster Comput., vol. 19, no. 4, pp. 2285-2293, 2016.

[13] X. Qi, et al., "A neutrosophic filter for high-density salt and pepper noise based on pixel-wise adaptive smoothing parameter," J. Vis. Comm. Image Represent, vol. 36, pp. 1-10, 2016.

[14] X. Zhang, et al., "Salt and pepper noise removal with image inpainting," AEU - Int. J. Electron. Commun, vol. 69, no. 1, pp. 307-313, 2015.

[15] S. Rajkumar and G. Malathi, "An Efficient Image Denoising Approach for the Recovery of Impulse Noise," Bulletin of Electrical Engineering and Informatics, vol. 6, no. 3, pp. 281-286, 2017.

[16] W. Jianwei, "A Noise Removal Algorithm of Color Image," TELKOMNIKA (Telecommunication Computing, Electronics and Control), vol. 12, no. 1, pp. 565-574, 2014.

[17] S. A. Ali, et al., "Salt and Pepper Noise Removal Using Resizable Window and Gaussian Estimation Function," International Journal of Electrical and Computer Engineering (IJECE), vol. 6, no. 5, pp. 2219-2224, 2016.

[18] D. Guo, et al., "Salt and pepper noise removal with noise detection and a patch-based sparse representation," Advances in Multimedia, 2014.

[19] X. Qu, et al., "Magnetic resonance image reconstruction from under sampled measurements using a patch-based nonlocal operator," Medical Image Analysis, vol. 18, no. 6, 2014.

[20] K. H. Jin and J. C. Ye, "Sparse and Low-Rank Decomposition of a Hankel Structured Matrix for Impulse Noise Removal," IEEE Transactions on Image Processing, vol. 27, no. 3, pp. 1448-1461, 2018.

[21] Y. Qin, "A new family of model-based impulsive wavelets and their sparse representation for rolling bearing fault diagnosis," IEEE Transactions on Industrial Electronics, vol. 65, no. 3, pp. 2716-2726, 2018. 